\title{
New Multi-Country Evidence on Purchasing Power Parity: Multi-Variate Unit Root Test Results
}

\author{
Jan J.J. Groen* \\ Tinbergen Institute, Erasmus University Rotterdam \\ Preliminary Draft - Not To Be Quoted Without Permission
}

\begin{abstract}
In this paper a likelihood-based multi-variate unit root testing framework is utilized to test whether the real exchange rates of G10 countries are non-stationary. The framework uses a likelihood ratio statistic which combines the information across all involved countries while retaining heterogeneous rates of mean reversion. This likelihood ratio statistic has an asymptotic distribution which can be typified as a summation of squared, univariate Dickey and Fuller (1979) distributions. Our multi-variate unit root tests indicate that bilateral G10 real exchange rates are stationary, irrespective of the numeraire country. On the other hand, the choice of the numeraire country seems to be of importance for the issue whether mean reversion rates across G10 real exchange rates are heterogeneous or homogeneous.
\end{abstract}

Keywords: Multi-variate unit root testing, maximum likelihood estimation, PPP, real exchange rates.

JEL classification: C12, C23, F31.

\section{Introduction}

Purchasing power parity (PPP) is a main building bloc for open-economy macroeconomic models and it implies that the competitiveness between two countries is equalized. A practical implication of PPP is that real exchange rates are stationary and it has given impetus to a whole literature on testing for stationary real exchange rates. In general, applying conventional augmented Dickey and Fuller (1979) (ADF) unit root tests on

*Tinbergen Institute, Erasmus University Rotterdam, P.O. Box 1738, NL-3000 DR Rotterdam, The Netherlands; E-mail: groen@few.eur.nl. 
real exchange rates relative to the United States (U.S.) could not reject the null of nonstationary real exchange rates. For example, Mark (1990) is not able to reject the null of non-stationarity for monthly real exchange rates relative to the U.S. and the United Kingdom (U.K.) for the 1973-1988 period whereas Papell (1997) has the same result for both monthly and quarterly U.S. real exchange rates over the 1973-1994 period. With respect to Germany-based real exchange rates both Mark (1990) and Papell (1997) provide more positive estimation results, albeit that they still do not significantly reject the hypothesis of non-stationarity for a majority of their real exchange rates. ${ }^{1}$

Since the Monte Carlo analysis in Shiller and Perron (1985) it is well known that the power of ADF unit root tests depend on the time span of the sample utilized in testing. As the time span of the post-Bretton Woods floating rate sample is rather short, 1973 up to the present, one can be doubtful that conventional ADF unit root tests are capable of detecting persistent, but stationary patterns in real exchange rates. One possible remedy for this problem is to look at panel data sets of real exchange rates. One can discern two groups of panel-based unit root tests of real exchange rates. Studies like Frankel and Rose (1996), MacDonald (1996), Oh (1996) and Papell (1997) have conducted panel unit root testing on real exchange rates using a version of the Levin and Lin (1992) panel unit root test. In general these studies find evidence for stationary real exchange rates in panels for 6 to 100 real exchange rates relative to both the U.S. and Germany on postBretton Woods samples. However, the evidence within panels of less than 10 countries is weak. Also, Papell (1997) fails to find evidence for stationarity within several samples of quarterly U.S.-based real exchange rates.

A major disadvantage of panel unit root testing based on the Levin and Lin (1992) approach is the assumption of cross-sectional independence between the different real exchange rates within the panel. Monte Carlo experiments in O'Connell (1998) indicate that panel unit root tests that neglect cross-sectional dependence yields severely biased test results on cross-sectionally correlated data. Given the fact that real exchange rates relative to the same base country are contemporaneously correlated, one should be doubtful with respect to test results based on Levin and Lin (1992) approach. A second group of panel-based studies, most notably Abuaf and Jorion (1990) and O'Connell (1998), utilize panel unit root test regressions where they allow for cross-sectional correlation across the included real exchange rates. On a monthly sample of G10 real exchange rates over the period 1973-1987 Abuaf and Jorion (1990) only rejects the null of non-stationarity marginally at a $10 \%$ significance level. O'Connell (1998) in panels of 12 to 64 countries with quarterly data over the period 1973-1995 cannot reject the null of non-stationary real exchange rates at all.

When properly conducted, that is allowing for cross-sectional dependence, panel unit root tests give mixed results on the issue whether or not real exchange rates are stationary. However, the bulk of panel-based studies are based on the assumption of identical rates of mean reversion and the weak panel-based evidence in favor of PPP could very well be caused by inappropriately assuming homogeneous speeds of mean reversion across

\footnotetext{
${ }^{1}$ Froot and Rogoff (1995) contains a detailed survey of time series-based empirical tests of PPP.
} 
countries, as suggested by O'Connell (1998, p. 18). For example, bilateral real exchange rates behave differently when monetary shocks in the bilateral relation ship are dominant than when real shocks are dominant. It is known from the literature that deviations from PPP are of short duration for high inflation countries. Also, when the home country has linked its monetary policy to that of the base country, based for example on a target zone regime, PPP deviations do not last long. On the other hand, when productivity growth in the home country is for a long period higher than in the base country Balassa (1964) argues that the corresponding bilateral real exchange rate will exhibit a sustained appreciation, implying a low rate of mean reversion. Finally, the mean reversion of real exchange rates can be slowed down by the existence of transportation costs (see Dumas 1992) and when these transportation costs differ across countries they could lead to differing speeds of adjustment. Hence, in order to profit from the extra information in multi-country samples it could be worthwhile to conduct multi-country tests of PPP based on crosssectional heterogeneity of mean reversion parameters.

Multi-country tests of PPP under parameter heterogeneity have up to now not been applied on a frequent basis. Coakley and Fuertes (1997) test the validity of PPP for U.S.-based real exchange rates of G10 countries over the 1973-1995 period within the heterogeneous panel unit root testing framework of Im et al. (1997)) and they can reject the null of non-stationary real exchange rates. But, the results of Coakley and Fuertes (1997) should be treated with suspicion as the Im et al. (1997) framework, like the Levin and Lin (1992) framework, is based on the assumption of cross-sectional independence. Hakkio (1984) does allow for cross-sectional dependence as he estimates a system of four U.S.-based real exchange rates with generalized least squares (GLS), and his estimation results does not provide evidence for PPP. However, the Hakkio (1984) results are not explicitly based on the non-stationarity of real exchange rates under the null and are therefore unreliable. The most reliable results available in case of heterogeneous panels are provided by Engel et al. (1997), who use dollarized price levels over the period 1978-1994 for two cities in each of the U.S., Canada, Germany and Switzerland. Engel et al. (1997) construct three panel models comprising intra-national real exchange rates, national real exchange rates and continental (North-America versus Europe) real exchange rates, and they simultaneously estimate these three panel models with GLS. Based on parametric bootstrap distributions they test if each of the three panels are composed of non-stationary real exchange rate data and these tests reject the validity of PPP. Yet, Engel et al. (1997) only allow for a limited degree of parameter heterogeneity: across the three panels there is heterogeneity and within each of the three panel models the mean reversion speeds are homogeneous. This particular specification could very well be the cause of their negative results on the PPP hypothesis.

As an alternative to existing studies, our paper proposes to estimate a system of $N$ ADF test regressions with iterative seemingly unrelated regression estimation (SURE) where the parameters differ for each equation. Likelihood ratio statistics are constructed to test the null hypothesis that all $N$ series are non-stationary versus the alternative hypothesis that all $N$ series are stationary. Compared to the existing literature our 
framework has several advantageous features. First, the set-up of our multi-variate unit root testing framework is such that it allows for different rates of mean reversion under the alternative of stationary series. Next, the estimates and tests within our likelihood-based framework are robust to any contemporaneous correlation across the series in our system. In fact, our likelihood-based framework actually utilizes the presence of contemporaneous correlation to enhance the power of the multi-variate unit root test. Existing studies of panel unit root tests on contemporaneously correlated data use (parametric) bootstrap distributions, as they claim that "...if there is cross-correlation in the data (...) the distributions of the statistics are not the same as before and are not known." (Maddala and $\mathrm{Wu}$ 1996, p. 14). Yet, for our multi-variate likelihood ratio unit root test we are able to determine the distribution even if the data are cross-correlated.

The multi-variate unit root test is used to test for the validity of PPP under crosssectional heterogeneity for G10 real exchange rates within the 1973-1996 post-Bretton Woods period. In contrast to the existing literature, we not only use the U.S. as the numeraire country. Both within pure time series data (Frenkel 1981, Mark 1990) and within panel data sets (Jorion and Sweeney 1996, Papell 1997) there is more evidence for stationary real exchange rates when instead of the U.S. Germany is used as the base country. Therefore, we use Germany as one of our base countries. Also, like Mark (1990) we use the United Kingdom (U.K.) as numeraire country. Finally, we use Japan as a base country for our G10 bilateral real exchange rates as this is the second largest nonEuropean country within the set of G10 countries and because the Japanese economy has undergone several structural changes during this period. The multi-variate unit root test results indicate that irrespective of the base country G10 bilateral real exchange rates are stationary. We also test whether mean reversion speeds are homogeneous across the G10 real exchange rates, and this does seem to depend upon the choice of numeraire country.

The remainder of this paper is organized as follows. In section 2 we provide a short overview of existing panel unit root tests. The likelihood-based multi-variate unit root testing framework is described in section 3. Multi-variate tests on the stationarity of G10 real exchange rates are reported in section 4 . Section 5 concludes the paper.

\section{Existing Panel Unit Root Tests}

In order to improve upon the negative results of standard time series unit root tests, unit root testing on real exchange rates has recently been conducted within panels of $N$ real exchange rates. Most studies base their analysis on the Levin and Lin (1992) framework which utilizes a test regression like ${ }^{2}$

$$
\Delta x_{i t}=\delta_{i}+\alpha x_{i, t-1}+\sum_{j=1}^{p} \gamma_{i j} \Delta x_{i, t-j}+\epsilon_{i t}, i=1, \ldots, N ; t=1, \ldots, T,
$$

\footnotetext{
${ }^{2}$ The most appropriate specification for unit root tests on real exchange rates is the specification with a constant included in the test regression.
} 
where $\Delta x_{i t}=x_{i t}-x_{i, t-1}, \delta_{i}$ is a constant which can differ across the cross-sections, $i$ is the cross-section index and $t$ is the time series index. Levin and Lin (1992) assume in (1) cross-sectionally unrelated disturbances: $\epsilon_{i t} \sim \mathrm{N}\left(0, \sigma_{i}^{2}\right)$ for $i=1, \ldots, N$, and $p$ lagged first differences are added to guarantee that the $\epsilon_{i t}$ 's are not autocorrelated. The non-stationarity of $x_{i t}$ for $i=1, \ldots, N$ can now be tested in (1) through a t-statistic $t_{\alpha}$ for $H_{0}: \alpha=0$ versus $H_{1}: \alpha<0$. Levin and $\operatorname{Lin}(1992)$ derive that for $T \rightarrow \infty, N \rightarrow \infty$ and $\sqrt{N} / T \rightarrow 0$ a proper transformation of $t_{\alpha}$ converges in the limit to a standard normal distribution: ${ }^{3}$

$$
\sqrt{1.25} t_{\alpha}+\sqrt{1.875} N \Rightarrow \mathrm{N}(0,1)
$$

A drawback of panel unit root testing based on (1), is the assumption of a homogeneous adjustment speed under the alternative hypothesis. Such an alternative hypothesis implies two things:

(a) $\alpha_{i}<0$ for $i=1, \ldots, N$;

(b) and conditional on (a): $\alpha_{1}=\cdots=\alpha_{N}$.

When in reality only (a) is valid, assuming a common $\alpha$ in (1) can be too restrictive and could decrease the power to reject the null in favor of a true alternative hypothesis. A possible solution is to base multi-country unit root testing of real exchange rates on the framework of $\mathrm{Im}$ et al. (1997). This framework is based on the estimation of the ADF test regression for each $x_{1 t}, \ldots, x_{N t}$ separately:

$$
\Delta x_{i t}=\delta_{i}+\alpha_{i} x_{i, t-1}+\sum_{j=1}^{p_{i}} \gamma_{i j} \Delta x_{i, t-j}+\epsilon_{i t}
$$

and constructing $N$ conventional ADF t-statistics $t_{\alpha, i}$ under the null $\alpha_{i}=0$ for $i=$ $1, \ldots, N$. Assuming $\operatorname{Cov}\left(\epsilon_{i t}, \epsilon_{j t}\right)=0$ for $i, j=1, \ldots, N$ with $i \neq j, \operatorname{Im}$ et al. (1997) propose to test $H_{0}: \alpha_{i}=0$ versus $H_{1}: \alpha_{i} \leq 0$ through

$$
\Pi_{\bar{t}}=\frac{\sqrt{N}\left(\bar{t}-E\left(t_{\alpha, i} \mid \alpha_{i}=0\right)\right)}{\sqrt{\operatorname{Var}\left(t_{\alpha, i} \mid \alpha_{i}=0\right)}} \Rightarrow \mathrm{N}(0,1),
$$

where $\bar{t}=\frac{1}{N} \sum_{i=1}^{N} t_{\alpha, i}$ and the asymptotic distribution is valid for $N \rightarrow \infty$ and $T \rightarrow \infty$. In (4) $\left.E\left(t_{\alpha, i} \mid \alpha_{i}=0\right)\right)$ and $\operatorname{Var}\left(t_{\alpha, i} \mid \alpha_{i}=0\right)$ are the cross-sectional mean and variance of the $t_{\alpha, i}$ 's under the null which are calculated through Monte Carlo simulations.

Both the Levin and Lin (1992) and the Im et al. (1997) approaches suffer from a number disadvantages which makes them inappropriate for testing the empirical validity of PPP across $N$ real exchange rates. Firstly, both approaches are based on cross-sectional independence between the involved real exchange rates and we argued before that this is a very unlikely assumption. As a consequence the asymptotic distributions in (2)

\footnotetext{
${ }^{3} \mathrm{~A}$ symbol " $\Rightarrow$ " indicates convergence in distribution.
} 
and (4) are invalid. Also, the set of testable hypotheses within the Im et al. (1997) framework are economically invalid. The Im et al. (1997) framework tests $H_{0}: \alpha_{i}=0$ versus $H_{1}: \alpha_{i} \leq 0$ for $i=1, \ldots, N$ and as such the alternative hypothesis is consistent with non-stationarity in a sub-sample of cross-sections. As the PPP hypothesis implies that each real exchange rate in the sample is stationary, the Im et al. (1997) hypothesis set-up is not compatible with the PPP hypothesis as one could still reject the null while a fraction of the real exchange rates are non-stationary. In the next section we propose an alternative framework, which allows for both heterogeneous rates of mean reversion and cross-sectional dependence. In contrast to the Im et al. (1997) approach, our multi-variate framework can be used to test the null of non-stationary real exchange rates versus the alternative that all $N$ real exchange rates are stationary.

\section{A Multi-Variate Framework for Unit Root Testing}

In this section we propose a likelihood-based framework in which we simultaneously test for non-stationarity across $N$ series. We first discuss in section 3.1 the involved estimation issues. Next, we construct in section 3.2 our multi-variate likelihood ratio unit root test statics and discuss the corresponding asymptotic distribution. Results of a Monte Carlo analysis of our test statistics can be found in section 3.3 .

\subsection{Maximum Likelihood Estimation}

In order to conduct a unit root test on an individual variable $x_{t}$ one can run a ADF test regression

$$
\Delta x_{t}=\delta z_{t}+\alpha x_{t-1}+\gamma w_{p t}+\epsilon_{t} ; t=1, \ldots, T
$$

In (5) $\Delta x_{t}=x_{t}-x_{t-1}$, the $m \times 1$ deterministic components vector $z_{t}$ either contains a constant: $z_{t}=1$, or a constant plus a linear time trend: $z_{t}=\left(\begin{array}{ll}1 & t\end{array}\right)^{\prime}$ with the $1 \times m$ coefficient vector $\delta$, and $w_{p t}=\left(\Delta x_{t-1} \cdots \Delta x_{t-p}\right)^{\prime}$ with the $1 \times p$ coefficient vector $\gamma$. The unit root test is a test if in (5) $\alpha=0$.

To conduct unit root testing on a variable $x_{i t}$ of the $i^{\text {th }}$ individual within a panel of $N$ individuals, we can stack $N$ ADF regressions like (5) into one system,

$$
\begin{aligned}
\Delta X_{t} & =\left(\begin{array}{c}
\delta_{1} \\
\vdots \\
\delta_{N}
\end{array}\right) z_{t}+\left(\begin{array}{ccc}
\alpha_{1} & 0 \cdots 0 & 0 \\
0 & \ddots & 0 \\
0 & 0 \cdots 0 & \alpha_{N}
\end{array}\right) X_{t-1}+\left(\begin{array}{ccc}
\gamma_{1} & 0 \cdots 0 & 0 \\
0 & \ddots & 0 \\
0 & 0 \cdots 0 & \gamma_{N}
\end{array}\right) W_{p t}+\varepsilon_{t} \\
& =\bar{\delta} z_{t}+\Phi X_{t-1}+\Gamma W_{p t}+\varepsilon_{t}
\end{aligned}
$$

where $\alpha_{i}$ relates $\Delta x_{i t}$ to $x_{i, t-1}$ and $\gamma_{i}$ relates $\Delta x_{i t}$ to $\Delta x_{i, t-1}, \ldots, \Delta x_{i, t-p_{i}}{ }^{4}$ The model in (6) consists of the $N \times 1$ vectors $X_{t-1}=\left(x_{1, t-1} \cdots x_{N, t-1}\right)^{\prime}, \Delta X_{t}=X_{t}-X_{t-1}$ and

\footnotetext{
${ }^{4}$ Note that the number of lagged first differences can differ across the equations of (6).
} 
$\varepsilon_{t}=\left(\epsilon_{1 t}^{\prime} \cdots \epsilon_{N t}^{\prime}\right)^{\prime}$, and the $\left(\sum_{i=1}^{N} p_{i}\right) \times 1$ vector $W_{p t}=\left(w_{p_{1} t, 1}^{\prime} \cdots w_{p_{N} t, N}^{\prime}\right)^{\prime}$ for $t=1, \ldots, T$ and $i=1, \ldots, N$. In (6) $z_{t}, x_{i t}, w_{p, i}, \delta_{i}, \alpha_{i}$ and $\gamma_{i}$ have an identical definition as in (5) for $i=1, \ldots, N$, and the coefficient matrices $\bar{\delta}, \Phi$ and $\Gamma$ have dimensions equal to $N \times m$, $N \times N$ and $N \times\left(\sum_{i=1}^{N} p_{i}\right)$ respectively. We assume a multivariate normal distribution for the disturbance vector $\varepsilon_{t}: \varepsilon_{t} \sim \mathrm{N}\left(\mathbf{0}_{N}, \Omega\right)$, with the $N$-dimensional vector of zeros $\mathbf{0}_{N}$ and the $N \times N$ covariance matrix structure,

$$
\Omega=\left(\begin{array}{ccc}
\omega_{11} & \cdots & \omega_{1 N} \\
\vdots & \ddots & \vdots \\
\omega_{N 1} & \cdots & \omega_{N N}
\end{array}\right)
$$

In $(7) \omega_{i j} \equiv \operatorname{Cov}\left(\epsilon_{i t}, \epsilon_{j t}\right)$ for $i, j=1, \ldots, N$.

Unit root testing across $N$ individuals simultaneously within the restricted vector autoregressive (VAR) model (6) involves testing the parameter restriction $\alpha_{1}=\cdots=$ $\alpha_{N}=0$. The VAR model subject to general exclusion restrictions is adopted in (6) in order to get an estimable model while still retaining a form of inter-dependency between the individuals through the non-diagonal structure (7) of the disturbance covariance matrix. Our restricted VAR hinges on the following assumption:

Assumption 3.1 There is no linear dependence between the variable $x_{i t}$ of individual $i$ and lags of the variable $x_{j t}$ of individual $j$ for $i \neq j$.

Proper estimation of the restricted VAR model (6) involves the usage of feasible GLS (or SURE), see Lütkepohl (1993, Section 5.2).

The log-likelihood function for model (6) can be written as, ${ }^{5}$

$$
\begin{aligned}
\ell(\bar{\delta}, \Phi, \Gamma, \Omega)=-\frac{N T}{2} \ln (2 \pi)+\frac{T}{2} \ln \left|\Omega^{-1}\right| \\
\quad-\frac{1}{2} \operatorname{tr}\left(\Omega^{-1}\left(\Delta X-Z \bar{\delta}^{\prime}-X_{-1} \Phi^{\prime}-W_{p} \Gamma^{\prime}\right)^{\prime}\left(\Delta X-Z \bar{\delta}^{\prime}-X_{-1} \Phi^{\prime}-W_{p} \Gamma^{\prime}\right)\right)
\end{aligned}
$$

where $\bar{\delta}, \Phi$ and $\Gamma$ are defined in (6) and $\Omega$ has an identical structure as (7). The $T \times N$ matrices $\Delta X, X_{-1}$ and the $T \times\left(\sum_{i=1}^{N} p_{i}\right)$ matrix $W_{p}$ in (8) can be defined as:

$$
\Delta X=\left(\begin{array}{c}
\Delta X_{1}^{\prime} \\
\vdots \\
\Delta X_{T}^{\prime}
\end{array}\right), X_{-1}=\left(\begin{array}{c}
X_{0}^{\prime} \\
\vdots \\
X_{T-1}^{\prime}
\end{array}\right) \text { and } W_{p}=\left(\begin{array}{c}
W_{p 1}^{\prime} \\
\vdots \\
W_{p T}^{\prime}
\end{array}\right)
$$

and the $T \times m$ matrix $Z$ equals $Z=\iota_{T}$ or $Z=(\iota \tau)$ with $\iota_{T}$ is a $T \times 1$ vector of ones and the $T \times 1$ vector $\tau=(1 \cdots T)^{\prime}$.

Maximum likelihood estimates of $\bar{\delta}, \Phi, \Gamma$ and the disturbance covariance matrix $\Omega$ in (6) can be obtained through iterative SURE (ISURE). Essential for this ISURE procedure

\footnotetext{
${ }^{5}$ The determinant of $\Omega^{-1}$ is indicated with $\left|\Omega^{-1}\right|$ and the trace of a matrix is indicated with $\operatorname{tr}(\cdots)$.
} 
is proper estimation of $\Omega$, and based on (8) $\Omega$ is estimated with the standard conditional maximum likelihood estimator: ${ }^{6}$

$$
\hat{\Omega}(\hat{\bar{\delta}}, \hat{\Phi}, \hat{\Gamma})=\frac{1}{T}\left(\Delta X-Z \hat{\bar{\delta}}^{\prime}-X_{-1} \hat{\Phi}^{\prime}-W_{P} \hat{\Gamma}^{\prime}\right)^{\prime}\left(\Delta X-Z \hat{\bar{\delta}}^{\prime}-X_{-1} \hat{\Phi}^{\prime}-W_{P} \hat{\Gamma}^{\prime}\right) .
$$

The ISURE procedure starts off with a consistent initial estimate of $\Omega$ :

$$
\hat{\Omega}\left(\hat{\Phi}_{\mathrm{OLS}}\right)=\left(\hat{\Omega}_{i j}\right)_{i, j=1, \ldots, N} \text { with } \hat{\Omega}_{i j}=\frac{1}{T} \sum_{t=1}^{T} \hat{\epsilon}_{i t} \hat{\epsilon}_{j t}^{\prime} .
$$

In (10) $\hat{\epsilon}_{i t}$ and $\hat{\epsilon}_{j t}$ are residuals resulting from $N$ OLS regressions of $\Delta x_{i t}$ on $z_{t}, x_{i, t-1}$ and $\Delta x_{i, t-1}, \ldots, \Delta x_{i, t-p}$ as in (5). The initial estimate (10) is used to estimate $\bar{\delta}, \Phi$ and $\Gamma$ through SURE and these SURE estimates in turn can be used to construct a new estimate of $\Omega$ based on (9). Next, we can construct new SURE estimates of $\bar{\delta}$, $\Phi$ and $\Gamma$ using the estimate of $\Omega$ based on the old SURE estimates of $\bar{\delta}, \Phi$ and $\Gamma$. Magnus (1978) shows that iterating in this manner until convergence of the estimators yields maximum likelihood estimates of $\bar{\delta}, \Phi, \Gamma$ and $\Omega$.

\subsection{Multi-Variate Unit Root Testing}

For unit root testing across $N$ individuals simultaneously based on a specification like (6), we make use of SURE estimators as outlined in section 3.1. Hence, we can only consider the cases where $T>N$ and the limiting behaviour of our test statistics are based on large $T$ asymptotics while assuming a fixed cross section dimension $N$.

As the null hypothesis of $N$ unit roots involves a restriction on $N$ parameters simultaneously, we shall use a likelihood ratio test to test for non-stationarity in our SURE system. One can straightforwardly show that the maximized value of (8) conditional on the maximum likelihood estimates $\hat{\bar{\delta}}, \hat{\Phi}$ and $\hat{\Gamma}$ in combination with disturbance covariance matrix estimator (9) can be specified as $^{7}$

$$
\ell_{\max }[\hat{\bar{\delta}}, \hat{\Phi}, \hat{\Gamma}, \hat{\Omega}(\hat{\bar{\delta}}, \hat{\Phi}, \hat{\Gamma})]=\ell_{\max }^{1}=-\frac{N T}{2}(1+\ln (2 \pi))-\frac{T}{2} \ln |\hat{\Omega}(\hat{\bar{\delta}}, \hat{\Phi}, \hat{\Gamma})|
$$

Under the unit root restriction, i.e. $\alpha_{1}=\cdots=\alpha_{N}=0$ in (6), maximum likelihood estimation is identical as in section 3.1 but without $x_{1, t-1}, \ldots, x_{N, t-1}$ included in our restricted VAR model. The corresponding maximized log-likelihood function equals:

$$
\ell_{\max }[\hat{\bar{\delta}}, \hat{\Gamma}, \hat{\Omega}(\hat{\bar{\delta}}, \hat{\Gamma})]=\ell_{\max }^{0}=-\frac{N T}{2}(1+\ln (2 \pi))-\frac{T}{2} \ln |\hat{\Omega}(\hat{\bar{\delta}}, \hat{\Gamma})|
$$

\footnotetext{
${ }^{6}$ The number of time series $T$ is identical for each equation as this greatly simplifies the estimation of covariance matrix $\Omega$. Hence, we consider in this paper only systems with balanced times series observations.

${ }^{7}$ Note that $\ln \left|\Omega^{-1}\right|=-\ln |\Omega|$.
} 
The likelihood ratio test statistic for $H_{0}: \alpha_{1}=\cdots=\alpha_{N}=0$ within (6) versus $H_{1}: \alpha_{i} \neq 0$ for $i=1, \ldots, N$ is now identical to:

$$
\mathrm{LR}_{\Phi=0}=2\left(\ell_{\max }^{1}-\ell_{\max }^{0}\right)=T[\ln |\hat{\Omega}(\hat{\bar{\delta}}, \hat{\Gamma})|-\ln |\hat{\Omega}(\hat{\bar{\delta}}, \hat{\Phi}, \hat{\Gamma})|]
$$

The asymptotic behaviour of the multi-variate unit root test statistic in (13) can be typified as

\section{Proposition 3.1 Let,}

(a) the estimates of $\delta_{1}, \ldots, \delta_{N}, \alpha_{1}, \ldots, \alpha_{N}, \gamma_{1}, \ldots, \gamma_{N}$ and $\Omega$ be fully converged estimates from the iterative estimation schemes of section 3.1 both under the null hypothesis $\left(\alpha_{1}=\cdots=\alpha_{N}=0\right)$ and the alternative hypothesis,

(b) each of the $N$ series $x_{1 t}, \ldots, x_{N t}$ be $I(1)$,

(c) the cross-section dimension $N$ be fixed and the time series dimension $T \rightarrow \infty$.

Then the limiting distribution of $L R_{\Phi=0}$ in (13) equals:

$$
L R_{\Phi=0} \Rightarrow \sum_{i=1}^{N}\left[\left(\int \check{B}_{i} d B_{i}\right)^{2}\left(\int \check{B}_{i}^{2}\right)^{-1}\right] .
$$

In (14) " $\Rightarrow$ " denotes convergence in distribution, $B_{i}(u)$ is a scalar standard Brownian motion for individual $i$ on the interval $u \in[0,1], \int \check{B}_{i} d B_{i} \equiv \int_{0}^{1} \check{B}_{i}(u) d B_{i}(u) d u$ and $\check{B}_{i}(u)=B_{i}(u)$ if in $(6) \delta_{1}=\cdots=\delta_{N}=0$ or $\check{B}_{i}(u)=\bar{B}_{i}(u)$. When appropriate, $\bar{B}_{i}(u)$ equals for individual $i \bar{B}_{i}(u)=B_{i}(u)-\int_{0}^{1} B(u) d u$ if in $(6) z_{t}=1$ or $\bar{B}_{i}(u)=B_{i}(u)-a_{i}-b_{i} t$ if in $(6) z_{t}=\left(\begin{array}{ll}1 & t\end{array}\right)$ with $a_{i}$ and $b_{i}$ resulting from regressing $B_{i}(u)$ on a constant and $a$ linear time trend.

Proof: See Appendix A.

Expression (14) is identical to a summation of $N$ squared Dickey and Fuller (1979) limiting distributions for the univariate ADF unit root test. Appendix B describes how we compute the critical values for test statistic (13) based on the asymptotic distributions from proposition 3.1 .

The finite sample properties of test statistic (13) can be improved through a degrees of freedom correction as suggested by Sims (1980). It involves replacing $T$ in (13) by the average degrees of freedom per equation under the alternative hypothesis:

$$
\mathrm{CLR}_{\Phi=0}=(T-d)[\ln |\hat{\Omega}(\hat{\bar{\delta}}, \hat{\Gamma})|-\ln |\hat{\Omega}(\hat{\bar{\delta}}, \hat{\Phi}, \hat{\Gamma})|]
$$

where $^{8}$

$$
d=\frac{1}{N}\left(N(m+1)+\sum_{i=1}^{N} p_{i}\right) .
$$

\footnotetext{
${ }^{8}$ The number of deterministic components per equation equals $m(m=0, m=1$ or $m=2$ ), the number of lagged first differences per equation equals $p_{j}$ and we have 1 lagged level $x_{i, t-1}$ per equation.
} 
Obviously, the corrected likelihood ratio test statistic (15) has smaller values than (13) and in finite samples combined with a large number of parameters CLR $_{\Phi=0}$ could very well be much closer to the asymptotic distribution (14) than $\mathrm{LR}_{\Phi=0}$.

\subsection{Monte Carlo Evaluation}

To study the behaviour of our multi-variate unit root test statistics (13) and (15) we conduct a Monte Carlo analysis on artificial samples with comparable dimensions as the multi-country systems used in section 4 . We report the rejection frequencies both for a true null hypothesis and a true alternative hypothesis at a nominal size of $5 \%$, based on 6,000 Monte Carlo experiments.

Within the Monte Carlo experiments the data generating process (DGP) of the artificial series $y_{i t}$ used in our tests equals:

$$
y_{i t}=c_{i}+\rho_{i} y_{i, t-1}+\mu_{i t}, \quad i=1, \ldots, N ; t=1, \ldots, T .
$$

A specification with a constant is chosen in (16) as we use this specification in section 4 and it is the most appropriate one for testing the PPP hypothesis. Also, we set $T=96$ in (16) which is comparable to the number of quarterly observations within the 1973-1996 sample used in section 4 . The cross-section dimension is set at $N=9$ as we have 9 real exchange rates in the multi-country systems of section 4 . We also set $N=3,6$ so that we can determine how the sizes and power ratios react to increases in the number of variables analyzed within the SURE system. The innovations $\mu_{i t}$ in (16) are generated through

$$
\mu_{i t}=\lambda_{i} \mu_{i, t-1}+\epsilon_{i t}
$$

where $\left(\epsilon_{1 t} \cdots \epsilon_{N t}\right)^{\prime} \sim \mathrm{N}\left(\mathbf{0}_{N}, \Sigma\right)$ with $^{9}$

$$
\Sigma=\Theta^{\prime} \Theta, \quad \Theta \text { is } N \times N \text { and } \Theta \sim \mathrm{U}(0,1) .
$$

Randomly generating the elements of the $\Theta$ matrix in (18) from an uniform distribution $\mathrm{U}(0,1)$ guarantees that the $\epsilon_{i t}$ 's in $(17)$ are positively cross-correlated, as in the historical samples from section 4 .

Sizes and power ratios are computed both with and without first order serially correlated $\mu_{i t}$ 's in (17):

Size without serial correlation: for $i=1, \ldots, N$ we have in (16) $c_{i}=0$ and $\rho_{i}=1$, and in (17) $\lambda_{i}=0$.

Size with serial correlation: for $i=1, \ldots, N$ we have in (16) $c_{i}=0$ and $\rho_{i}=1$, and in $(17) \lambda_{i} \sim \mathrm{U}(-0.5,0.5)$.

Power without serial correlation: for $i=1, \ldots, N$ we have in $(16) c_{i} \sim \mathrm{U}(-1,1)$ and $\rho_{i} \sim \mathrm{U}(0.9,1)$, and in (17) $\lambda_{i}=0$.

\footnotetext{
${ }^{9}$ The denomination $\mathrm{U}\left(k_{1}, k_{2}\right)$ indicates that we draw from an uniform distribution on the interval between, but NOT including, $k_{1}$ and $k_{2}$.
} 
Power with serial correlation: for $i=1, \ldots, N$ we have in $(16) c_{i} \sim \mathrm{U}(-1,1)$ and $\rho_{i} \sim \mathrm{U}(0.9,1)$, and in $(17) \lambda_{i} \sim \mathrm{U}(-0.5,0.5)$.

For the power computations we have chosen to draw the mean reversion parameters from $\mathrm{U}(0.9,1)$ in order to have an ample amount of heterogeneity, comparable with the range of estimated parameters in section 4, combined with a significant degree of persistence. All other parameters were also drawn from uniform distributions for each $i=1, \ldots, N$ so that we have heterogeneity across the $N$ cross-sections. As a benchmark we also calculate the sizes and power ratios for the univariate ADF unit root test, based on the above mentioned DGP's only now with $N=1$.

The results of the Monte Carlo experiments on our multi-variate unit root tests are reported in table 1 . When we have no serially correlated innovations we see that both the $\mathrm{LR}_{\Phi=0}$ and the $\mathrm{CLR}_{\Phi=0}$ statistics have a correct size at the $95 \%$ quantile from distribution (14). In case of first order serially correlated innovations we have fitted our SURE system (6) with a common lag order $p$ equal to 1,2 and 3 . For $p=1$ we have again in all cases a correct size. When the utilized lag order increases from 1 to 2 and 3 we see in table 1 that at $N=9$ the $\mathrm{LR}_{\Phi=0}$ statistics has a tendency to slightly overreject the true null hypothesis. The $\mathrm{CLR}_{\Phi=0}$ statistic, however, retains a correct size when at $N=9$ the lag order increases to 2 and 3. Overall, the $\mathrm{CLR}_{\Phi=0}$ statistic has better a size than the $\mathrm{LR}_{\Phi=0}$ statistic when the number of parameters increase substantially.

When we look at the power ratios in table 1 we see that at $N=3$ we have for both multi-variate unit root test statistics power ratios in the range of $60 \%-72 \%$. When we compare this to the univariate ADF test it becomes clear that already at very moderate cross-section dimensions our $\mathrm{LR}_{\Phi=0}$ and $\mathrm{CLR}_{\Phi=0}$ statistics have a superior power performance relative to univariate tests. An increase in the number of series from 3 to 6 and 9 results in a substantial increase in the power ratios to levels beyond the $90 \%$ value. Given these results it would be interesting to determine how much of the high power ratios of our multi-variate unit root test statistics is due to the presence of positive cross-correlated innovations. Therefore, we also compute power ratios based on Monte Carlo experiments without contemporaneous correlation in the data, i.e. we set the off-diagonal elements of $\Sigma$ in (18) equal to 0 . When the $\mathrm{LR}_{\Phi=0}$ and $\mathrm{CLR}_{\Phi=0}$ statistics are based on non-correlated data the corresponding power ratios, reported in square brackets in table 1 , are considerably lower than in the case of correlated data. The presence of positive cross-correlated innovations results in at least a $65 \%$ increase of the power of our multi-variate unit root test statistics.

To further assess the importance of contemporaneously correlated data, we have also employed our Monte Carlo experiments to compute the sizes and power ratios of the Im et al. (1997) panel unit root test. In section 2 we stated that when we have crosscorrelated data the corresponding limiting distribution of the $\operatorname{Im}$ et al. test is incorrect. This is confirmed by the fact that in table 1 the $\operatorname{Im}$ et al. test at $N=6$ and $N=9$ is considerably oversized. Corresponding power ratios in table 1 indicate that the $\operatorname{Im}$ et al. test has a low power relative to the $\mathrm{LR}_{\Phi=0}$ and $\mathrm{CLR}_{\Phi=0}$ statistics, despite the fact that the $\operatorname{Im}$ et al. test is oversized under the null. This is caused by the fact that within the 
Im et al. framework the $N$ test regressions are separately estimated and not jointly as in our framework. Therefore, next to the objections already mentioned in section 2 the power calculations suggest another objection to the usage of the Im et al. test on real exchange rate data: a relative lack of power in case of a moderate number of persistent, stationary series. Hence, our likelihood-based multi-variate unit root test statistics are the most appropriate for a multi-country analysis of real exchange rates.

\section{New Multi-Country Unit Root Test Results of PPP}

In this section we apply the multi-variate unit root testing framework from section 3 on the real exchange rates of the G10 countries in order to test the validity of PPP for all these countries. Section 4.1 contains an description of the data. Also, we conduct in this subsection univariate unit root tests on bilateral G10 real exchange rates relative to the U.S., Germany Japan and the U.K. Next, we report in section 4.2 multi-variate unit root test results for our four sets of G10 bilateral real exchange rates.

\subsection{The Data and Univariate Unit Root Test Results}

In its logarithmic form the real exchange rate for the home country versus a foreign country is defined as

$$
q=e+p^{*}-p,
$$

where $q, e, p^{*}$ and $p$ are the logarithm of the real exchange rate, the nominal exchange rate, the foreign aggregate price level and the home aggregate price level respectively. Long-run PPP is valid when the real exchange rate has a constant mean through time, implying an equalized relative competitiveness in the long-run between two countries. Thus $q$ in (19) must be stationary, i.e. one should reject the null hypothesis

$$
H_{0}: \Delta q_{t}=\epsilon_{t}, \epsilon \sim \text { i.i.d. }\left(0, \sigma^{2}\right) ; t=1, \ldots, T,
$$

in favor of the alternative hypothesis

$$
H_{1}: \Delta q_{t}=\delta+\alpha q_{t-1}+\epsilon_{t}, \alpha<0 .
$$

An intercept $\delta$ is included in (21) to correct for measurement errors due to the fact that we use in practice price indices and not actual price levels. Note that (21) allows for short-run deviations from PPP.

We consider real exchange rates for 10 of the most important industrialized countries (G10), i.e. Canada, France, Germany, Italy, Japan, The Netherlands, Sweden, Switzerland, the U.K. and the U.S. Quarterly observations from 1973.1 through 1996.4 are used in the estimation of our systems of real exchange rates. Logarithms of real exchange rates are constructed as in (19), where we use the consumer price index (CPI) as a proxy of the aggregate price level. Data on the CPI's and exchange rates are obtained from the IMF's International Financial Statistics (IFS). ${ }^{10} \mathrm{G} 10$ real exchange rates are constructed

\footnotetext{
${ }^{10}$ The CPI data are from IFS line code 64 .
} 
relative to four numeraire countries: the U.S., Germany, Japan and the U.K. In constructing real exchange rates relative to the U.S. we use quarterly average U.S. dollar exchange rates as the CPI data are also quarterly averages. ${ }^{11}$ In case of real exchange rates relative to Germany, Japan and the U.K., the nominal exchange rates are calculated through cross-rates based on the U.S. dollar exchange rates.

To get a feel of the degree of persistence within bilateral G10 real exchange rates, we conduct univariate ADF unit root tests for G10 real exchange rates relative to our four base countries. We use the ADF unit root test with a constant included in the test regression, that is we conduct a t-test for $\alpha=0$ in

$$
\Delta q_{t}=\delta+\alpha q_{t-1}+\sum_{j=1}^{p} \gamma_{j} \Delta q_{t-j}+\epsilon_{t}
$$

The lag order for the ADF test regressions is selected as follows. First, we determine an optimal lag order through the Akaike Information Criterion (AIC), based on a comparison of AIC criteria computed for lag orders ranging from 0 to 8 in (22). Next, we used Lagrange-Multiplier (LM) serial correlation tests at 1, 4 and 8 lags to determine whether the residuals of (22) at the optimal AIC lag order are white noise. If that is not the case, we increase the lag order until the LM serial correlation tests indicate that the residuals of (22) are indeed white noise.

From table 2 it becomes clear that irrespective of the base country univariate unit root tests are in general not able to reject the null of non-stationary real exchange rates. The ADF tests for Germany-based real exchange rates provide the most favorable evidence for the PPP hypothesis, as we can reject the null of non-stationarity for France, The Netherlands and Switzerland. For the other base countries we are only able to reject the null of noon-stationary real exchange rates in case of the real exchange rate of Switzerland relative to the U.K. The estimated measures of mean reversion seems to have more favorable values when we use Germany and the U.K. as the base countries. All things considered, the results in table 2 indicate that even if real exchange rates are stationary their degree of persistence is such that univariate unit root tests are not able reject the null of non-stationarity.

\subsection{Multi-Variate Unit Root Test Results}

The failure of univariate unit root tests to reject in section 4.1 the null of non-stationary real exchange rates could be due to slow rates of mean reversion such that one only can find evidence for stationarity within samples of data with a long time span. One possible solution is the usage of panel techniques described in section 2 , but these techniques are based on the possibly invalid assumption of homogeneous cross-country rates of mean reversion. As an alternative we apply in this subsection the multi-variate framework of section 3. The power analysis in section 3.3 indicates that when we have cross-correlated

\footnotetext{
${ }^{11}$ The exchange rate data are from IFS line code "rf".
} 
data, our multi-variate framework has ample power to reject the null when the data are stationary but persistent in nature. On average the cross-correlations of the relative changes in real exchange rates with respect to the U.S., Germany, Japan and the U.K. equals $0.56,0.33,0.69$ and 0.61 respectively. Hence, a priori we would expect that our multi-variate unit root test statistics yield more positive results with respect to the PPP hypothesis than the univariate unit root tests, especially for the base countries the U.S., Japan and the U.K.

For G10 bilateral real exchange rates relative to the U.S., Germany, Japan and the U.K. we conduct ISURE estimation on a system like (6) with $z_{t}=1$, i.e.

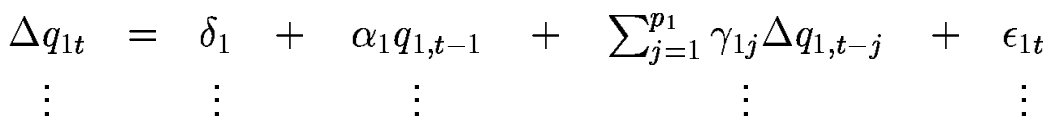

$$
\begin{aligned}
& \Delta q_{N t}=\delta_{N}+\alpha_{N} q_{N, t-1}+\sum_{j=1}^{p_{N}} \gamma_{N j} \Delta q_{N, t-j}+\epsilon_{N t}
\end{aligned}
$$

and $N=9$. Next, after we have estimated (23) under the restriction $\alpha_{1}=\cdots=\alpha_{N}=0$ we construct our likelihood ratio unit root test statistics (13) and (15). We analyze (23) both with individual specific lag orders and a common lag order, i.e. $p_{1}=\cdots=p_{N}=p$. For the case of heterogeneous lag orders we use in each equation of system (23) the same lag order as in the corresponding equation in table $2 .{ }^{12}$ In case of a common lag order the optimal lag order $p$ in (23) equals the lag order at the minimum of the AIC criteria for the whole system computed over the range $p=0, \ldots, 5$. An upper bound lag order equal to 5 is chosen as this is the maximum lag order in the univariate test regressions in table 2. The selected common lag orders are reported in table 3.

The results of our multi-variate unit root tests for our G10 bilateral real exchange rates based on a common lag order are summarized in table 3. The likelihood-based test statistics are able to reject the null of non-stationary real exchange rates for all four sets of G10 real exchange rates. When Germany and Japan are used as the numeraire country we can reject the null comfortably at the $1 \%$ significance level. In case of the base countries the U.S. and the U.K. the p-values of the test statistics indicate that we easily can reject the null at the $5 \%$ significance level. Test results based on individual specific lag orders in (23) can be found in table 4, and we see that the usage of a heterogeneous lag order do not significantly change the results for the rates relative to Germany; again we reject at very low significance levels. With respect to the U.S., Japan and the U.K. the uncorrected $\mathrm{LR}_{\Phi=0}$ statistic indicates a rejection of the null at the $5 \%$ significance level, whereas the results for the $\mathrm{CLR}_{\Phi=0}$ statistic indicates a rejection at the $10 \%$ significance level with p-values very close to $5 \%$.

Although the usage of individual specific lag orders does not seem to influence the strength of rejections of the null in case Germany-based real exchange rates, this seems to be the case for the other sets of real exchange rates especially relative to Japan. The Monte Carlo experiments in section 3.3 showed that at $N=9$ the $\mathrm{LR}_{\Phi=0}$ statistic becomes slightly oversized when the total number of parameters becomes large. This result could

\footnotetext{
${ }^{12}$ For the validity of this approach, see Lütkepohl (1993, pp. 182-183)
} 
explain the difference in tables 3 and 4 for the p-values of the rates relative to the U.S., Japan and the U.K., as the number of parameters in case of a common lag order is larger than for the heterogeneous lag order (see table 2). On the other hand, we saw in section 3.3 that the $\mathrm{CLR}_{\Phi=0}$ statistic still has a correct size when we have a large amount of parameters and the results in table 3 based on the CLR $\mathrm{C}_{\Phi=0}$ statistic are for the rates relative to the U.S., Japan and the U.K. not different than for the $\mathrm{LR}_{\Phi=0}$ statistic. Moreover, the selection procedure for a common lag order in (23) is based on system-wide information in contrast to the selection of the heterogeneous lag orders which are based on country-specific information only. Therefore, the common lag order selection procedure could very well provide extra information with respect to the dynamic behaviour of the real exchange rates relative to the U.S., Japan and the U.K. resulting in the lower p-values for these base countries in table 3 compared to table 4 . Given this and the fact that the rejections in table 4 are still quite strong, we can safely say that for all our G10 real exchange rates we have strong evidence for stationary real exchange rates.

In tables 3 and 4 the cross-country variability of maximum likelihood estimates for the mean reversion coefficients seems to depend on the choice of the numeraire country. Even though this cross-country variability is low when the U.S. and the U.K. are the base countries, for real exchange rates relative to Germany and Japan the mean reversion speeds per country are quite heterogeneous in nature. For example, rough measures for the half life of shocks ${ }^{13}$ indicate that in table 3 Germany-based rates of Canada, Japan and the U.S. have a half-life somewhere between 14 and 19 quarters, whereas France, The Netherlands, Sweden and the U.K. exhibit a half-life between 3 and 7 quarters. Note also that for the real exchange rates relative to both Germany and Japan we have the result that the adjustment speeds relative to European countries are much higher than with respect to non-European countries.

It could therefore be worthwhile to test whether the mean reversion rates $\alpha_{i}$ are homogeneous across the equations of (23). To achieve this we construct likelihood ratio statistics for the null hypothesis $\alpha_{1}=\cdots=\alpha_{N}$, where the likelihood ratio statistic has under the null a $\chi^{2}(8)$ distribution. We also calculate AIC criteria for both heterogeneous and homogeneous $\alpha_{i}$ 's in (23), where the minimum of these AIC criteria indicates which case is empirically more appropriate. From the lower parts in tables 3 and 4 we see that both the likelihood ratio statistic and the AIC criteria confirm our suspicions: mean reversion rates for the base countries Germany and Japan are heterogeneous, while for rates relative to the U.S. and the U.K. the $\alpha_{i}$ 's are identical for all countries. Note that estimates for the common $\alpha_{\mathrm{HOM}}$ are identical or close to -0.06 for both U.S.-based and U.K.-based real exchange rates. Apparently, shocks to the numeraire countries U.S. and U.K. dominated all other shocks resulting in a common adjustment speed.

\footnotetext{
${ }^{13}$ These measures equal $\ln (0.5) / \ln \left(1+\hat{\alpha}_{i}\right)$.
} 


\section{Conclusions}

The validity of long-run PPP implies that real exchange rates are stationary, i.e. in time real exchange rates revert back to a constant mean. This paper proposes and employs a multi-variate framework for unit root testing in multi-country systems of real exchange rates, while retaining cross-country differences in mean reversion rates. By treating the panel of data explicitly as a restricted, high dimensional VAR model we are able to derive appropriate estimation and testing methods based on the corresponding log-likelihood function. Utilizing time series-based asymptotics in combination with a fixed cross-section dimension enables us to derive limiting distributions which are also applicable when the series in the panel are contemporaneously correlated. Monte Carlo experiments for systems with empirically sensible dimensions show that our multi-variate unit root test statistic behaves well both under a true null of non-stationarity and under a true alternative of stationarity, especially when a degrees-of-freedom correction is employed. The Monte Carlo results indicate that our multi-variate unit root test is not only robust to crosscorrelations in the data, the usage of cross-correlated data also improves the power of the test significantly.

Our multi-variate unit root testing framework tests the null that all series in the panel are non-stationary versus the alternative that all series are stationary. Because of this set-up our framework is more appropriate for testing the validity of long-run PPP than other available heterogeneous panel unit root tests. The empirical tests are conducted on the bilateral real exchange rates of 10 large industrialized (G10) countries. We construct four sets of G10 real exchange rates relative to four different numeraire countries. For all the multi-country systems our multi-variate approach is able to reject the null of nonstationary real exchange rates, both with a common lag order in the multi-country systems and with country-specific lag orders. Test results with respect to the issue whether mean reversion rates are homogeneous across countries yield conclusions which depend on the choice of the base country. Real exchange rates relative to the U.S. and the U.K. exhibit homogeneous adjustment speeds, whereas mean reverting patterns within real exchange rates relative to Germany and Japan are highly heterogeneous in nature.

Homogeneous adjustment speeds in case of U.S.-based and U.K.-based real exchange rates suggest that in these cases shocks in the base country seemed to have dominated all other shocks. While in the case of the U.S. this is not surprising given the large relative size of its economy, it is more puzzling for U.K.-based rates. Maybe the "boom-and-bust" behaviour of British monetary policy in the 1970s and 1980s lead to such an amount of economic variability that it dominated all foreign shocks. Also, our system-based estimates for the base countries Germany and Japan suggests an asymmetric pattern in mean reversion speeds: very high with respect to European countries and very low with respect to non-European countries. It would be interesting for future research to assess which part monetary and real shocks play in this asymmetric pattern. A further research topic is to apply the framework of this paper on real exchange rates based on disaggregated price data, e.g. city-based price indexes or sector-based prices. Finally, based on an appropriately restricted disturbance covariance matrix our framework could 
be extended to the case where we have both a large number of cross-sections and time series observations.

\section{Appendix}

\section{A Proof of Proposition 3.1}

In the following proofs we discard the presence of lagged first differences in (6), and we assume that we have $\gamma_{1}=\cdots=\gamma_{N}=0$ combined with a vector of disturbances $\varepsilon_{t}$ which does not exhibit serial correlation. From Dickey and Fuller (1979) and Said and Dickey (1984) we know that the inclusion of lagged first differences within ADF test regressions, in order to guarantee white noise innovations, does not influence the asymptotic behaviour of the ADF t-statistic relative to the case of no higher order dynamics. Johansen (1991) has an identical result in the case of likelihood ratio cointegration rank statistics within unrestricted VAR models of non-stationary variables. As (6) can both be considered as a system of $N$ ADF test regressions and as a restricted VAR model of $N$ non-stationary variables, $L R_{\Phi=0}$ is under the null asymptotically identical whether or not $\gamma_{1}=\cdots=\gamma_{N}=$ 0 in (6) as long as we have white noise disturbances. Hence, for notational convenience we base all our proofs on the absence of higher order dynamics in (6). Also, our proofs are at first based on the absence of deterministic components in (6) but we discuss at the end of this Appendix the extension to the case of deterministic components.

In deriving the limiting behaviour of $\mathrm{LR}_{\Phi=0}$ we make use of the following results:

1. We make use of the properties of "vec"-operators and Kronecker-product operators as summarized in Lütkepohl (1993, Appendix A.11 and A.12), we use in particular:

$$
\begin{aligned}
\operatorname{vec}(A B C) & =\left(C^{\prime} \otimes A\right) \operatorname{vec}(B), \\
(A \otimes B)(C \otimes D) & =(A C \otimes B D),
\end{aligned}
$$

where $A, B$ and $C$ are appropriate matrices and "vec" denotes vectorization of a matrix by stacking the columns of this matrix.

2. For $T \rightarrow \infty$ we have (see Hamilton 1994, chapters 17 and 18):

$$
\begin{aligned}
\frac{1}{T^{2}} X_{-1}^{\prime} X_{-1} & \Rightarrow \Omega^{\frac{1}{2}}\left(\int W_{N} W_{N}^{\prime}\right) \Omega^{1^{\prime}} \\
\frac{1}{T} X_{-1}^{\prime} \Delta X & \Rightarrow \Omega^{\frac{1}{2}}\left(\int W_{N} d W_{N}^{\prime}\right) \Omega^{\frac{1}{2}^{\prime}} .
\end{aligned}
$$

In (A.2) $W_{N}(u)=\left(B_{1}(u) \cdots B_{N}(u)\right)^{\prime}$ is a $N$-dimensional vector Brownian Motion with covariance matrix $I_{N}$ and $u \in[0,1], B_{i}(u)$ is a scalar standard Brownian Motion, $\int W_{N} d W_{N}^{\prime} \equiv \int_{0}^{1} W_{N}(u) d W_{N}(u) d u$, and $\Omega$ is the true non-diagonal disturbance covariance matrix as in (7). Note that " $\Rightarrow$ " indicates convergence in distribution, whereas in the remainder of this Appendix " $\stackrel{p}{\rightarrow}$ " indicates convergence in probability. 


\section{The Proof}

For $\delta_{1}=\cdots=\delta_{N}=0, \gamma_{1}=\cdots=\gamma_{N}=0$ and no serial correlation within the innovations vector $\varepsilon_{t}$ in (6), log-likelihood function (8) can also be written as

$$
\begin{aligned}
\ell(\Phi, \Omega)=-\frac{N T}{2} \ln (2 \pi)+ & \frac{T}{2} \ln \left|\Omega^{-1}\right| \\
& -\frac{1}{2} \operatorname{vec}\left(\Delta X-X_{-1} \Phi\right)^{\prime}\left(\Omega^{-1} \otimes I_{T}\right) \operatorname{vec}\left(\Delta X-X_{-1} \Phi\right),
\end{aligned}
$$

with the $T \times T$ identity matrix $I_{T}$. We can write within the last part of log-likelihood function (A.3)

$$
\begin{aligned}
\operatorname{vec}\left(\Delta X-X_{-1} \Phi\right)= & \\
& \operatorname{vec}(\Delta X)-\operatorname{vec}\left(X_{1,-1} \alpha_{1} \ldots X_{N,-1} \alpha_{N}\right) \\
& =\operatorname{vec}(\Delta X)-\left(I_{N} \otimes X_{-1}\right) F_{\Phi}\left(\begin{array}{c}
\alpha_{1} \\
\vdots \\
\alpha_{N}
\end{array}\right),
\end{aligned}
$$

where $I_{N}$ is a $N \times N$ identity matrix and $F_{\Phi}$ is a $N^{2} \times N$ selection matrix,

$$
F_{\Phi}=\left(\begin{array}{cccc}
e_{1} & \mathbf{0}_{N} & \cdots & \mathbf{0}_{N} \\
\mathbf{0}_{N} & e_{2} & & \mathbf{0}_{N} \\
\mathbf{0}_{N(N-3)} & \mathbf{0}_{N(N-3)} & \ddots & \mathbf{0}_{N(N-3)} \\
\mathbf{0}_{N} & \mathbf{0}_{N} & & e_{N}
\end{array}\right)=\left(\left(e_{1} \otimes e_{1}\right) \cdots\left(e_{N} \otimes e_{N}\right)\right) .
$$

In (A.5) $e_{i}$ is the $i^{\text {th }}$ column of the identity matrix $I_{N}$ and $\mathbf{0}_{s}$ is a $s$-dimensional column vector of zeros with $s=N$ or $N(N-3)$. Substituting (A.4) in log-likelihood (A.3) and maximizing (A.3) with respect to $\Phi$ given $\Omega$, yields the following estimator of $\alpha_{1}, \ldots, \alpha_{N}$ :

$$
\left(\begin{array}{c}
\hat{\alpha}_{1} \\
\vdots \\
\hat{\alpha}_{N}
\end{array}\right)=\left(F_{\Phi}^{\prime}\left(\Omega^{-1} \otimes X_{-1}^{\prime} X_{-1}\right) F_{\Phi}\right)^{-1} F_{\Phi}^{\prime}\left(\Omega^{-1} \otimes I_{T}\right) \operatorname{vec}\left(X_{-1}^{\prime} \Delta X\right),
$$

which is a SURE estimator. The conditional maximum likelihood estimator of $\Omega$ given the estimate $\hat{\Phi}$ equals:

$$
\hat{\Omega}(\hat{\Phi})=\frac{1}{T}\left(\Delta X-X_{-1} \hat{\Phi}\right)^{\prime}\left(\Delta X-X_{-1} \hat{\Phi}\right) .
$$

Using (A.6) and (A.7) in the ISURE procedures from section 3.1 yields maximum likelihood estimates but Magnus (1978) has shown that the estimates after one iteration have the same asymptotic distribution as fully converged estimates. In the following we make use of this property of the one-step SURE estimator. 
Based on log-likelihood function (A.3) for both the $\alpha_{1}=\cdots=\alpha_{N}=0$ and $\alpha_{i} \neq 0$ specifications, and substituting the estimates $\hat{\alpha}_{1}, \ldots, \hat{\alpha}_{N}$ from (A.6) and the true disturbance covariance matrix $\Omega$, we can write $\operatorname{LR}_{\Phi=0}=2[\ell(\hat{\Phi}, \Omega)-\ell(\Omega)]$ as

$$
\begin{aligned}
\operatorname{LR}_{\Phi=0}= & 2[\ell(\hat{\Phi}, \Omega)-\ell(\Omega)] \\
= & \operatorname{vec}(\Delta X)^{\prime}\left(\Omega^{-1} \otimes I_{T}\right) \operatorname{vec}(\Delta X) \\
& \quad-\operatorname{vec}\left(\Delta X-X_{-1} \hat{\Phi}\right)^{\prime}\left(\Omega^{-1} \otimes I_{T}\right) \operatorname{vec}\left(\Delta X-X_{-1} \hat{\Phi}\right) \\
= & \operatorname{vec}\left(X_{-1} \hat{\Phi}\right)^{\prime}\left(\Omega^{-1} \otimes I_{T}\right) \operatorname{vec}\left(X_{-1} \hat{\Phi}\right) .
\end{aligned}
$$

Under $H_{0}: \alpha_{1}=\cdots=\alpha_{N}=0, \hat{\Omega}(\hat{\Phi})$ in (A.7) is a consistent estimate of $\Omega$. Hence, given (A.8) and $\hat{\Omega}=\hat{\Omega}(\hat{\Phi}) \stackrel{p}{\rightarrow} \Omega$ we have

$$
\begin{aligned}
\operatorname{LR}_{\Phi=0} \simeq & \operatorname{vec}\left(X_{-1} \hat{\Phi}\right)^{\prime}\left(\hat{\Omega}^{-1} \otimes I_{T}\right) \operatorname{vec}\left(X_{-1} \hat{\Phi}\right) \\
& =\left(\begin{array}{c}
\hat{\alpha}_{1} \\
\vdots \\
\hat{\alpha}_{N}
\end{array}\right)^{\prime} F_{\Phi}^{\prime}\left(\hat{\Omega}^{-1} \otimes X_{-1}^{\prime} X_{-1}\right) F_{\Phi}\left(\begin{array}{c}
\hat{\alpha}_{1} \\
\vdots \\
\hat{\alpha}_{N}
\end{array}\right) \\
& =\left(F_{\Phi} \operatorname{vec}\left(X_{-1}^{\prime} \Delta X \hat{\Omega}^{-1}\right)\right)^{\prime}\left[F_{\Phi}^{\prime}\left(\hat{\Omega}^{-1} \otimes X_{-1}^{\prime} X_{-1}\right) F_{\Phi}\right]^{-1} F_{\Phi}^{\prime} \operatorname{vec}\left(X_{-1}^{\prime} \Delta X \hat{\Omega}^{-1}\right),
\end{aligned}
$$

where $F_{\Phi}$ is defined in (A.5) and the third expression results from substituting estimator (A.6).

Based on $\hat{\Omega}=\hat{\Omega}(\hat{\Phi}) \stackrel{p}{\rightarrow} \Omega,($ A.1), (A.2) and the continous mapping theorem, we have for $T \rightarrow \infty$ :

$$
\begin{aligned}
\frac{1}{T^{2}}\left[F_{\Phi}^{\prime}\left(\hat{\Omega}^{-1} \otimes X_{-1}^{\prime} X_{-1}\right) F_{\Phi}\right]^{-1} \Rightarrow & {\left[F_{\Phi}^{\prime}\left(\Omega^{-1} \otimes \Omega^{\frac{1}{2}} \int W_{N} W_{N}^{\prime} \Omega^{2^{\prime}}\right) F_{\Phi}\right]^{-1} } \\
& =\left[F_{\Phi}^{\prime}\left(\Omega^{-\frac{1}{2}} \otimes \Omega^{\frac{1}{2}}\right)\left(I_{N} \otimes \int W_{N} W_{N}^{\prime}\right)\left(\Omega^{-\frac{1}{2}} \otimes \Omega^{\frac{1}{2}}\right)^{\prime} F_{\Phi}\right]^{-1},
\end{aligned}
$$

and

$$
\begin{aligned}
\frac{1}{T} F_{\Phi}^{\prime} \operatorname{vec}\left(X_{-1}^{\prime} \Delta X \hat{\Omega}^{-1}\right) \Rightarrow & F_{\Phi}^{\prime} \operatorname{vec}\left(\Omega^{\frac{1}{2}} \int W_{N} d W_{N}^{\prime} \Omega^{-\frac{1^{\prime}}{2}}\right) \\
= & F_{\Phi}^{\prime}\left(\Omega^{-\frac{1}{2}} \otimes \Omega^{\frac{1}{2}}\right) \operatorname{vec}\left(\int W_{N} d W_{N}^{\prime}\right)
\end{aligned}
$$

In order to manipulate the expressions in (A.10) and (A.11) we define the following:

$$
\begin{aligned}
\Omega^{-\frac{1}{2}} & =\left(\Psi_{1}^{\prime} \cdots \Psi_{N}^{\prime}\right)^{\prime}, \quad \text { with } \Psi_{i} \text { is } 1 \times N, \\
\Omega^{\frac{1}{2}} & =\left(\Upsilon_{1}^{\prime} \cdots \Upsilon_{N}^{\prime}\right)^{\prime}, \quad \text { with } \Upsilon_{i} \text { is } 1 \times N
\end{aligned}
$$


Utilizing (A.12) we can now write

$$
\begin{aligned}
F_{\Phi}^{\prime}\left(\Omega^{-\frac{1}{2}} \otimes \Omega^{\frac{1}{2}}\right)\left(\Omega^{-\frac{1}{2}} \otimes \Omega^{\frac{1}{2}}\right)^{\prime} F_{\Phi} & =\left(\begin{array}{c}
\Psi_{1} \otimes \Upsilon_{1} \\
\vdots \\
\Psi_{N} \otimes \Upsilon_{N}
\end{array}\right)\left(\begin{array}{c}
\Psi_{1} \otimes \Upsilon_{1} \\
\vdots \\
\Psi_{N} \otimes \Upsilon_{N}
\end{array}\right)^{\prime} \\
& =\left(\begin{array}{ccc}
\left(\Psi_{1} \Psi_{1}^{\prime}\right)\left(\Upsilon_{1} \Upsilon_{1}^{\prime}\right) & \cdots & \left(\Psi_{1} \Psi_{N}^{\prime}\right)\left(\Upsilon_{1} \Upsilon_{N}^{\prime}\right) \\
\vdots & \ddots & \vdots \\
\left(\Psi_{N} \Psi_{1}^{\prime}\right)\left(\Upsilon_{N} \Upsilon_{1}^{\prime}\right) & \cdots & \left(\Psi_{N} \Psi_{N}^{\prime}\right)\left(\Upsilon_{N} \Upsilon_{N}^{\prime}\right)
\end{array}\right) \\
& =P P^{\prime},
\end{aligned}
$$

where $P P^{\prime}$ is the Choleski decomposition of the $N \times N$ matrix in the second right hand side expression in (A.13). Using (A.13), pre-multiplying the expression within square brackets in (A.10) with $P^{-1}$ and post-multiplying with $P^{-1^{\prime}}$ yields

$$
\begin{aligned}
& {\left[P^{-1} F_{\Phi}^{\prime}\left(\Omega^{-\frac{1}{2}} \otimes \Omega^{\frac{1}{2}}\right)\left(I_{N} \otimes \int W_{N} W_{N}^{\prime}\right)\left(\Omega^{-\frac{1}{2}} \otimes \Omega^{\frac{1}{2}}\right)^{\prime} F_{\Phi} P^{-1^{\prime}}\right]^{-1} } \\
& \left.=\left(\begin{array}{ccc}
\left(\Psi_{1} \Psi_{1}^{\prime}\right)\left(\Upsilon_{1} \int W_{N} W_{N}^{\prime} \Upsilon_{1}^{\prime}\right) & \cdots & \left(\Psi_{1} \Psi_{N}^{\prime}\right)\left(\Upsilon_{1} \int W_{N} W_{N}^{\prime} \Upsilon_{N}^{\prime}\right) \\
\vdots & \ddots & \vdots \\
\left(\Psi_{N} \Psi_{1}^{\prime}\right)\left(\Upsilon_{N} \int W_{N} W_{N}^{\prime} \Upsilon_{1}^{\prime}\right) & \cdots & \left(\Psi_{N} \Psi_{N}^{\prime}\right)\left(\Upsilon_{N} \int W_{N} W_{N}^{\prime} \Upsilon_{N}^{\prime}\right)
\end{array}\right) P^{-1^{\prime}}\right)^{-1} \\
& =\left(\begin{array}{ccc}
\int B_{1}^{2} & 0 \cdots 0 & 0 \\
0 & \ddots & 0 \\
0 & 0 \cdots 0 & \int B_{N}^{2}
\end{array}\right) .
\end{aligned}
$$

For (A.11) we have, based on (A.1) and (A.13), the following result:

$$
\begin{aligned}
P^{-1} F_{\Phi}^{\prime}\left(\Omega^{-\frac{1}{2}} \otimes \Omega^{\frac{1}{2}}\right) \operatorname{vec}\left(\int W_{N} d W_{N}^{\prime}\right) & =P^{-1}\left(\begin{array}{c}
\Upsilon_{1} \int W_{N} d W_{N}^{\prime} \Psi_{1}^{\prime} \\
\vdots \\
\Upsilon_{N} \int W_{N} d W_{N}^{\prime} \Psi_{N}^{\prime}
\end{array}\right) \\
& =\left(\begin{array}{c}
\int B_{1} d B_{1} \\
\vdots \\
\int B_{N} d B_{N}
\end{array}\right)
\end{aligned}
$$

Substitution of (A.14) and (A.15) in (A.9) results in the following limiting expression for $\mathrm{LR}_{\Phi=0}$ :

$$
\begin{aligned}
\operatorname{LR}_{\Phi=0} & \Rightarrow\left(\begin{array}{c}
\int B_{1} d B_{1} \\
\vdots \\
\int B_{N} d B_{N}
\end{array}\right)^{\prime}\left(\begin{array}{ccc}
\int B_{1}^{2} & 0 \cdots 0 & 0 \\
0 & \ddots & 0 \\
0 & 0 \cdots 0 & \int B_{N}^{2}
\end{array}\right)^{-1}\left(\begin{array}{c}
\int B_{1} d B_{1} \\
\vdots \\
\int B_{N} d B_{N}
\end{array}\right) \\
& =\sum_{i=1}^{N}\left[\left(\int B_{i} d B_{i}\right)^{2}\left(\int B_{i}^{2}\right)^{-1}\right] .
\end{aligned}
$$




\section{Deterministic Components}

We can concentrate log-likelihood function (8) with respect to the deterministic components through OLS regressions of the elements of $\Delta X_{t}, X_{t-1}$ and $W_{p t}$ on the deterministic component vector $z_{t}$, as $z_{t}$ has an identical content for each equation of (6). ${ }^{14}$ Hence, we have after adjusting for the effect of the deterministic components:

$$
\begin{aligned}
\Delta \tilde{X}_{i} & =M_{Z} \Delta X_{i} \text { and } \Delta \tilde{X}=\left(\Delta \tilde{X}_{1} \cdots \Delta \tilde{X}_{N}\right), \\
\tilde{X}_{i,-1} & =M_{Z} X_{i,-1} \text { and } \tilde{X}_{-1}=\left(\tilde{X}_{1, t-1} \cdots \tilde{X}_{N,-1}\right), \\
\tilde{W}_{p, i} & =M_{Z} W_{p, i} \text { and } \tilde{W}_{p}=\left(\tilde{W}_{p, 1} \cdots \tilde{W}_{p, N}\right),
\end{aligned}
$$

with $M_{Z}=I_{T}-Z\left(Z^{\prime} Z\right)^{-1} Z^{\prime}$. Replacing $\Delta X, Z, X_{-1}$ and $W_{p}$ with the variables of (A.16) in the ISURE procedure from section 3.1 yields therefore identical maximum likelihood estimates of $\Phi$ and $\Gamma$ in (6) as in the original ISURE procedure. Under the null of $N$ non-stationary variables, i.e. $\delta_{1}=\cdots=\delta_{N}=\alpha_{1}=\cdots=\alpha_{N}=0$, we now have $\bar{B}_{i}(u)=B_{i}(u)-\int_{0}^{1} B_{i}(u) d u$ or $\bar{B}_{i}(u)=B_{i}(u)-a_{i}-b_{i} t$, and $d B_{i}(u)-\int_{0}^{1} d B_{i}(u) d u=d B_{i}(u)$ or $d B_{i}(u)-a_{i}-b_{i} t=d B_{i}(u){ }^{15}$ Hence, we replace in all relevant formulae of the previously described proof $W_{N}(u)$ with $\bar{W}_{N}(u)=\left(\bar{B}_{1}(u) \cdots \bar{B}_{N}(u)\right)^{\prime}$ while retaining $d W_{N}$.

\section{B Critical Values}

The asymptotic distribution of our multi-variate likelihood ratio unit root test, as summarized in proposition 3.1, is a functional of Brownian Motions. As these are continuous time variables, one has to rely in practice on approximations to get proper critical values for our multi-variate unit root tests. Nielsen (1997) observes that within a single equation model the asymptotic behaviour of a likelihood ratio unit root test is very well approximated by a Gamma-distribution, especially for quantiles $\geq 50 \%$. The limiting distribution of a likelihood ratio unit root test within the single equation framework equals a squared Dickey and Fuller (1979) distribution and the limiting distribution in proposition 3.1 equals a summation of $N$ squared Dickey-Fuller distributions. Hence, we use a Gamma-distribution to approximate our asymptotic distributions.

The Gamma-distribution can be written as

$$
\Gamma(z ; r, a)=\int_{0}^{z} \frac{a^{r}}{\Gamma(r)} x^{r-1} \exp (-a x) d x, \quad z>0, r>0, a>0,
$$

where $\Gamma(\cdot)$ is the Gamma-function. When we can find proper values for the parameters $a$ and $r$, we can use (B.1) to approximate the distribution of our test statistic $z$ under the null. Following Doornik (1998), we can calibrate (B.1) through

$$
\hat{a}=\frac{m}{v}, \quad \hat{r}=\frac{m^{2}}{v}
$$

\footnotetext{
${ }^{14}$ See also the Frisch-Waugh-Lovell theorem in Davidson and MacKinnon (1993, pp. 19-24).

${ }^{15}$ Parameters $a_{i}$ and $b_{i}$ results from regressing $B_{i}(u)$ on an intercept and a linear time trend.
} 
where $m$ is the approximated asymptotic mean of $z$ under the null and $v$ is the approximated asymptotic variance. Doornik (1998) shows in Monte Carlo experiments that the above described procedures yields very accurate approximations of the asymptotic distributions of multivariate cointegration tests, which basically are squared multivariate Dickey-Fuller distributions.

Proposition 3.1 indicates that the asymptotic distribution of our test statistic is a summation of $N$ independent, squared Dickey-Fuller distributions. Therefore, the mean and variance of these distributions equals $N$ times the mean and $N$ times the variance of a single squared Dickey-Fuller distribution. Thus, we first approximate the asymptotic mean and variance of a single squared Dickey-Fuller distribution through Monte Carlo simulations. In these simulations we generate a discrete time random walk with 2,000 observations, compute the Dickey-Fuller t-value and take the square of this t-value. We iterate 100,000 times and calculate the mean and variance across the 100,000 generated squared t-values. These exercises are repeated for specifications with a constant or a constant plus trend added to the test regression, where we use either a demeaned or a detrended random walk. Based on the Monte Carlo mean and variance we determine in table B.1 approximations for the mean and variance of our distributions. Using the values from table B.1 we can now determine the values of $r$ and $a$ in (B.1) through (B.2). The resulting calibrated Gamma-distribution can now be used to compute asymptotic critical values or p-values for our multivariate unit root test. ${ }^{16}$

Table B.1: Mean and variance of the limiting distributions of Proposition $3.1^{\mathrm{a}}$

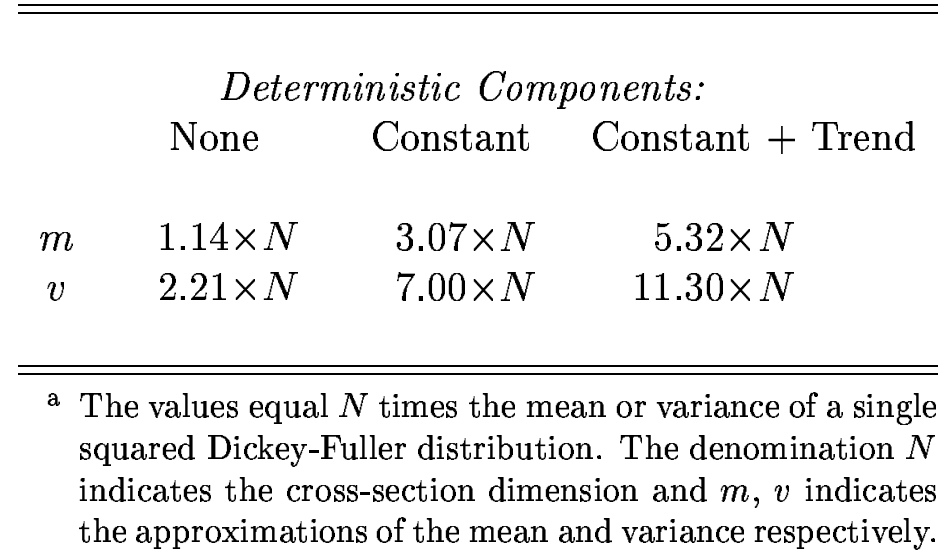

\footnotetext{
${ }^{16}$ A GAUSS procedure for calculating the $90 \%, 95 \%$ and $99 \%$ quantiles or p-values based on the fitted
} Gamma-distribution is available from the author. 


\section{References}

Abuaf, N. and P. Jorion, 1990, Purchasing Power Parity in the Long Run, Journal of Finance 44, 157-175.

Balassa, B., 1964, The Purchasing Power Parity Doctrine: A Reappraisal, Journal of Political Economy 72, 584-596.

Coakley, J. and A.M. Fuertes, 1997, New Panel Unit Root Tests of PPP, Economics Letters 57, 17-22.

Davidson, R. and J.G. MacKinnon, 1993, Estimation and Inference in Econometrics, Oxford University Press, Oxford.

Dickey, D.F. and W.A. Fuller, 1979, Distribution of the Estimators for Autoregressive Time Series with a Unit Root, Journal of the American Statistical Association 74, 427431.

Doornik, J.A., 1998, Approximations to the Asymptotic Distributions of Cointegration Tests, Journal of Economic Surveys 12, 573-593.

Dumas, B., 1992, Dynamic Equilibrium and the Real Exchange Rate in a Spatially Seperated World, Review of Financial Studies 5, 153-180.

Engel, C., M.K. Hendrickson and J.H. Rogers, 1997, Intra-National, Intra-Continental and Intra-Planetary PPP, Journal of the Japanese and International Economies 11, 480501.

Frankel, J.A. and A.K. Rose, 1996, A Panel Project on Purchasing Power Parity: Mean Reversion within and between Countries, Journal of International Economics 40, 209 224.

Frenkel, J., 1981, Flexible Exchange Rates, Prices and the Role of 'News': Lessons from the 1970s, Journal of Political Economy 89, 665-705.

Froot, K.A. and K. Rogoff, 1995, Perspectives on PPP and Long-Run Real Exchange Rates, in G. Grossman and K. Rogoff (editors), Handbook of International Economics, Vol. III, North Holland, Amsterdam.

Hakkio, C.S., 1984, A Re-examination of Purchasing Power Parity: A Multi-country and Multi-period Study, Journal of International Economics 15, 265-278.

Hamilton, J.D., 1994, Time Series Analysis, Princeton University Press, Princeton.

Im, K.S., M.H. Pesaran and Y. Shin, 1997, Testing for Unit Roots in Heterogeneous Panels, mimeo, Department of Applied Economics, Cambridge University. 
Johansen, S., 1991, Estimation and Hypothesis Testing of Cointegration Vectors in Gaussian Vector Autoregressive Models, Econometrica 59, 1551-1580.

Jorion, P. and R. Sweeney, 1996, Mean Reversion in Real Exchange Rates: Evidence and Implications for Forecasting, Journal of International Money and Finance 15, 535-550.

Levin, A. and Chien-Fu Lin, 1992, Unit Root Tests in Panel Data: Asymptotic and Finite Sample Properties, Discussion Paper 92-23, Department of Economics, University of California at San Diego.

Lütkepohl, H., 1993, Introduction to Multiple Time Series Analysis, 2nd edition, SpringerVerlag, Berlin.

MacDonald, R., 1996, Panel Unit Root Tests and Real Exchange Rates, Economics Letters $50,7-11$.

MacKinnon, J.G., 1991, Critical Values for Cointegration Tests, in R.F. Engle and C.W.J. Granger (editors), Long Run Economic Relationships: Readings in Cointegration, Oxford University Press, Oxford, pp. 267-276.

Maddala, G.S. and S. Wu, 1996, A Comparative Study of Unit Root Tests with Panel Data and a New Simple Test: Evidence from Simulations and the Bootstrap, mimeo, Department of Economics, Ohio State University.

Magnus, J.R., 1978, Maximum Likelihood Estimation of the GLS Model with Unknown Parameters in the Disturbance Covariance Matrix, Journal of Econometrics 7, 281-312.

Mark, N.C., 1990, Real Exchange Rates in the Long-Run: An Empirical Investigation, Journal of International Economics 28, 115-136.

Nielsen, B., 1997, Bartlett Correction of the Unit Root Test in Autoregressive Models, Biometrika 84, 500-504.

O'Connell, P., 1998, The Overvaluation of Purchasing Power Parity, Journal of International Economics 44, 1-19.

Oh, K., 1996, Purchasing Power Parity and Unit Root Tests Using Panel Data, Journal of International Money and Finance 15, 405-418.

Papell, D.H., 1997, Searching for Stationarity: Purchasing Power Parity Under the Current Float, Journal of International Economics 43, 313-332.

Said, S. and D.A. Dickey, 1984, Testing for Unit Roots in Autoregressive Moving-Average Models of Unknown Order, Biometrika 71, 599-607.

Shiller, R. and P. Perron, 1985, Testing the Random Walk Hypothesis: Power versus Frequency of Observation, Economics Letters 18, 381-386.

Sims, C.A., 1980, Macroeconomics and Reality, Econometrica 48, 1-48. 
Table 1: Size and power of the multi-variate unit root test with constant terms for a nominal size of $5 \% .^{\mathrm{a}}$

\begin{tabular}{|c|c|c|c|c|c|c|c|c|c|}
\hline & \multicolumn{3}{|c|}{$\underline{\text { No Serial Correlation }}$} & \multicolumn{6}{|c|}{$\underline{\text { Serial Correlation }}$} \\
\hline & & \multirow[b]{2}{*}{ Size } & \multirow[b]{2}{*}{ Power } & \multicolumn{2}{|c|}{$p=1$} & \multicolumn{2}{|c|}{$p=2$} & \multicolumn{2}{|c|}{$p=3$} \\
\hline & & & & Size & Power & Size & Power & Size & Power \\
\hline \multicolumn{10}{|c|}{ Augmented Dickey and Fuller (1979) Unit Root Test } \\
\hline & & 0.050 & 0.170 & 0.047 & 0.153 & 0.048 & 0.132 & 0.044 & 0.129 \\
\hline & & & Multi & riate $U$ & it Root & & & & \\
\hline \multirow[t]{5}{*}{$N=3$} & $\mathrm{LR}_{\Phi=0}$ & 0.052 & 0.718 & 0.058 & 0.693 & 0.054 & 0.669 & 0.056 & 0.647 \\
\hline & & & {$[0.267]$} & & {$[0.239]$} & & {$[0.216]$} & & {$[0.213]$} \\
\hline & $\mathrm{CLR}_{\Phi=0}$ & 0.047 & 0.707 & 0.046 & 0.669 & 0.042 & 0.640 & 0.040 & 0.606 \\
\hline & & & {$[0.245]$} & & {$[0.211]$} & & {$[0.176]$} & & {$[0.169]$} \\
\hline & IPS & 0.072 & 0.222 & 0.073 & 0.185 & 0.078 & 0.187 & 0.078 & 0.177 \\
\hline \multirow[t]{5}{*}{$N=6$} & $\mathrm{LR}_{\Phi=0}$ & 0.064 & 0.961 & 0.077 & 0.954 & 0.080 & 0.948 & 0.086 & 0.930 \\
\hline & & & {$[0.434]$} & & {$[0.417]$} & & {$[0.371]$} & & {$[0.362]$} \\
\hline & $\operatorname{CLR}_{\Phi=0}$ & 0.055 & 0.957 & 0.060 & 0.948 & 0.061 & 0.934 & 0.060 & 0.913 \\
\hline & & & {$[0.400]$} & & {$[0.372]$} & & {$[0.309]$} & & {$[0.291]$} \\
\hline & IPS & 0.136 & 0.378 & 0.142 & $0.330^{\circ}$ & 0.147 & 0.331 & 0.146 & 0.310 \\
\hline \multirow[t]{5}{*}{$N=9$} & $\mathrm{LR}_{\Phi=0}$ & 0.078 & 0.997 & 0.082 & 0.996 & 0.097 & 0.990 & 0.102 & 0.988 \\
\hline & & & {$[0.601]$} & & {$[0.575]$} & & {$[0.548]$} & & {$[0.501]$} \\
\hline & $\operatorname{CLR}_{\Phi=0}$ & 0.067 & 0.997 & 0.064 & 0.995 & 0.067 & 0.988 & 0.073 & 0.985 \\
\hline & & & {$[0.565]$} & & {$[0.518]$} & & {$[0.458]$} & & {$[0.412]$} \\
\hline & IPS & 0.182 & 0.472 & 0.179 & 0.429 & 0.184 & 0.419 & 0.189 & 0.392 \\
\hline \multicolumn{10}{|c|}{$\begin{array}{l}\text { The Monte Carlo experiments are based on } T=96 \text { and } 6,000 \text { simulations both with or without } \\
\text { first order serially correlated innovations, see the text. Denomination } p \text { indicates the used lag } \\
\text { order in (6). The statistics } L_{\Phi=0} \text { and } \mathrm{CLR}_{\Phi=0} \text { are defined in (13) and (15). Size and power } \\
\text { calculations are based on the asymptotic } 95 \% \text { quantile which in case of constant terms and } \\
N=3(N=6)[N=9] \text { equals } 17.807(30.211) \text { [41.848] (see Appendix B). Values within square } \\
\text { brackets are the power ratios computed when the data are not cross-correlated. The results for } \\
\text { the univariate ADF test are based on the appropriate } 5 \% \text { critical value from MacKinnon (1991). } \\
\text { Rows with "IPS" report the results for the Im et al. (1997) panel unit root test. }\end{array}$} \\
\hline
\end{tabular}




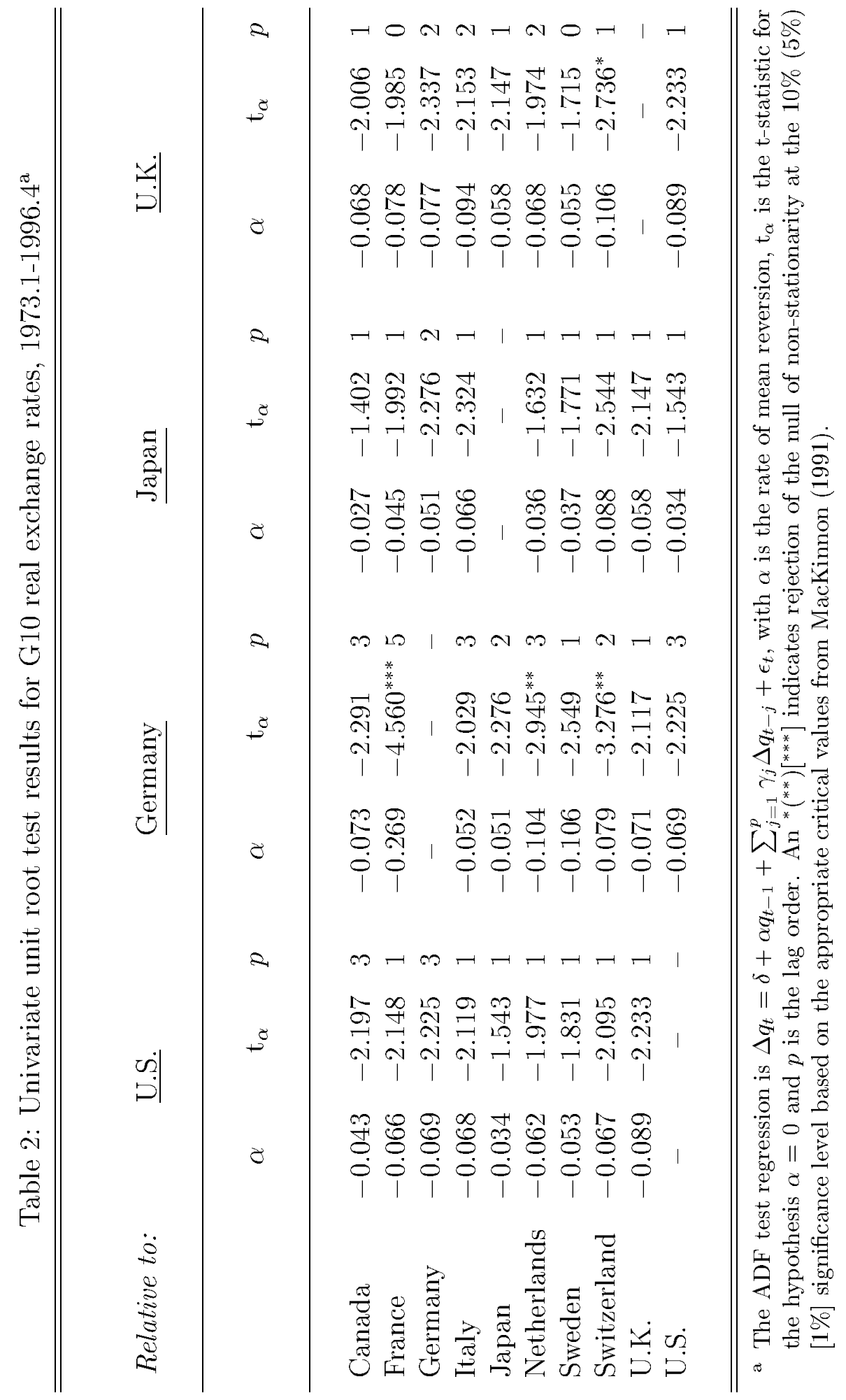


Table 3: Multi-variate unit root test results for G10 real exchange rates, $1973.1-1996.4^{\mathrm{a}}$

\begin{tabular}{|c|c|c|c|c|}
\hline Relative to: & U.S. & Germany & Japan & U.K. \\
\hline \multirow[t]{2}{*}{ Lag order $p$ : } & 3 & 4 & 3 & 3 \\
\hline & $\hat{\alpha}_{i}$ & $\hat{\alpha}_{i}$ & $\hat{\alpha}_{i}$ & $\hat{\alpha}_{i}$ \\
\hline Canada & -0.037 & -0.039 & -0.043 & -0.051 \\
\hline France & -0.067 & -0.196 & -0.083 & -0.056 \\
\hline Germany & -0.076 & - & -0.094 & -0.066 \\
\hline Italy & -0.081 & -0.051 & -0.100 & -0.093 \\
\hline Japan & -0.063 & -0.035 & -- & -0.057 \\
\hline Netherlands & -0.077 & -0.115 & -0.083 & -0.062 \\
\hline Sweden & -0.064 & -0.119 & -0.070 & -0.046 \\
\hline Switzerland & -0.084 & -0.066 & -0.127 & -0.086 \\
\hline U.K. & -0.054 & -0.095 & -0.089 & -- \\
\hline U.S. & 一 & -0.046 & -0.047 & -0.060 \\
\hline \multicolumn{5}{|c|}{ Likelihood Ratio Unit Root Tests } \\
\hline $\mathrm{LR}_{\Phi=0}$ & $\begin{array}{l}48.357 \\
(0.013)\end{array}$ & $\begin{array}{c}58.192 \\
(0.001)\end{array}$ & $\begin{array}{c}56.242 \\
(0.002)\end{array}$ & $\begin{array}{l}47.375 \\
(0.016)\end{array}$ \\
\hline $\mathrm{CLR}_{\Phi=0}$ & $\begin{array}{l}45.729 \\
(0.022)\end{array}$ & $\begin{array}{l}54.355 \\
(0.003)\end{array}$ & $\begin{array}{l}53.186 \\
(0.004)\end{array}$ & $\begin{array}{l}44.801 \\
(0.027)\end{array}$ \\
\hline \multicolumn{5}{|c|}{ Homogeneity Tests on $\alpha_{i}$} \\
\hline $\mathrm{LR}_{\mathrm{HOM}}$ & $\begin{array}{c}4.968 \\
{[0.761]}\end{array}$ & $\begin{array}{c}20.053 \\
{[0.010]}\end{array}$ & $\begin{array}{c}18.609 \\
{[0.017]}\end{array}$ & $\begin{array}{c}5.368 \\
{[0.718]}\end{array}$ \\
\hline $\mathrm{AIC}_{\mathrm{HOM}}$ & -66.632 & -65.573 & -66.556 & -66.676 \\
\hline $\mathrm{AIC}_{\mathrm{HET}}$ & -66.512 & -66.834 & -66.585 & -66.560 \\
\hline$\alpha_{\mathrm{HOM}}$ & -0.060 & - & -- & -0.060 \\
\hline
\end{tabular}

a ISURE estimates of $\alpha_{i}$ in (23) equal $\hat{\alpha}_{i}$. "LR $\mathrm{LR}_{\Phi=0}$ " and "CLR $\mathrm{CR}_{\Phi=0}$ " are likelihood ratio statistics for the null of $N$ unit roots, with the corresponding asymptotic p-values within parentheses (see Appendix B). Corresponding $90 \%, 95 \%$ and $99 \%$ quantiles are equal to $38.164,41.848$ and 49.359 respectively. " $\mathrm{LR}_{\mathrm{HOM}}$ " are likelihood ratio tests for the null $\alpha_{1}=\cdots=\alpha_{N}=\alpha_{\mathrm{HOM}}$, the square brackets contain corresponding p-values based on the $\chi^{2}(8)$ distribution, and " $\mathrm{AIC}_{\mathrm{HOM}}$ " ("AIC $\mathrm{HET}_{\mathrm{T}}$ ") is the AIC criterion in case of homogeneous (heterogeneous) $\alpha_{i}$ 's. 
Table 4: Multi-variate unit root test results for G10 real exchange rates with heterogeneous lag orders, 1973.1-1996.4 ${ }^{\mathrm{a}}$

$\begin{array}{lcccc}\text { Relative to: } & \text { U.S. } & \text { Germany } & \text { Japan } & \text { U.K. } \\ & & & & \\ & & & & \\ & \hat{\alpha}_{i} & \hat{\alpha}_{i} & \hat{\alpha}_{i} & \hat{\alpha}_{i} \\ & & & & \\ \text { Canada } & -0.040 & -0.047 & -0.033 & -0.043 \\ \text { France } & -0.065 & -0.238 & -0.066 & -0.048 \\ \text { Germany } & -0.071 & -- & -0.078 & -0.069 \\ \text { Italy } & -0.076 & -0.050 & -0.083 & -0.085 \\ \text { Japan } & -0.059 & -0.046 & -- & -0.062 \\ \text { Netherlands } & -0.072 & -0.091 & -0.061 & -0.054 \\ \text { Sweden } & -0.057 & -0.105 & -0.061 & -0.036 \\ \text { Switzerland } & -0.075 & -0.069 & -0.106 & -0.090 \\ \text { U.K. } & -0.058 & -0.085 & -0.070 & - \\ \text { U.S. } & - & -0.049 & -0.037 & -0.043\end{array}$

\section{Likelihood Ratio Unit Root Tests}

$\begin{array}{ccccc}\mathrm{LR}_{\Phi=0} & 42.412 & 56.610 & 42.501 & 42.315 \\ & (0.045) & (0.002) & (0.044) & (0.046) \\ \mathrm{CLR}_{\Phi=0} & 40.824 & 53.745 & 41.079 & 40.899 \\ & (0.061) & (0.004) & (0.058) & (0.060)\end{array}$

Homogeneity Tests on $\alpha_{i}$

$\begin{array}{crccc}\mathrm{LR}_{\mathrm{HOM}} & 2.852 & 18.090 & 17.298 & 9.021 \\ & {[0.943]} & {[0.021]} & {[0.027]} & {[0.341]} \\ & & & & \\ \mathrm{AIC}_{\mathrm{HOM}} & -65.997 & -66.755 & -65.774 & -65.933 \\ \mathrm{AIC}_{\mathrm{HET}} & -65.854 & -66.778 & -65.788 & -65.858 \\ \alpha_{\mathrm{HOM}} & -0.058 & - & - & -0.055\end{array}$

a See the notes of table 3 . The individual specific lag orders are identical to the lag orders of the corresponding equations in table 2. 